\title{
Blastocyst-endometrium interaction: intertwining a cytokine network
}

W.A. Castro-Rendón ${ }^{1,2}$

J.F. Castro-Álvarez ${ }^{1,2}$,

C. Guzmán-Martinez ${ }^{2}$ and J.C. Bueno-Sanchez ${ }^{1,2}$

\author{
${ }^{1}$ Semillero de Investigación SIMBIOSIS, \\ ${ }^{2}$ Grupo Reproducción - Corporación Biogénesis, \\ Universidad de Antioquia, Medellín, Colombia
}

\author{
Correspondence \\ W.A. Castro-Rendón \\ Grupo Reproducción \\ Sede de Investigación Universitaria \\ Cll 62 \#52-59, Laboratorio 534 \\ Universidad de Antioquia \\ Medellín \\ Colombia \\ Fax: +57-4-210-6470 \\ E-mail: castrowilson@gmail.com \\ $\ldots \ldots \ldots \ldots \ldots \ldots$.
}

Received November 3, 2005

Accepted June 21, 2006

\begin{abstract}
The successful implantation of the blastocyst depends on adequate interactions between the embryo and the uterus. The development of the embryo begins with the fertilized ovum, a single totipotent cell which undergoes mitosis and gives rise to a multicellular structure named blastocyst. At the same time, increasing concentrations of ovarian steroid hormones initiate a complex signaling cascade that stimulates the differentiation of endometrial stromal cells to decidual cells, preparing the uterus to lodge the embryo. Studies in humans and in other mammals have shown that cytokines and growth factors are produced by the pre-implantation embryo and cells of the reproductive tract; however, the interactions between these factors that converge for successful implantation are not well understood. This review focuses on the actions of interleukin-1, leukemia inhibitory factor, epidermal growth factor, heparin-binding epidermal growth factor, and vascular endothelial growth factor, and on the network of their interactions leading to early embryo development, peri-implantatory endometrial changes, embryo implantation and trophoblast differentiation. We also propose therapeutical approaches based on current knowledge on cytokine interactions.
\end{abstract}

\section{Introduction}

Some decades ago cytokines were known as intercellular messengers with a unique function in the immune system. Subsequently, experimental evidence showed that they participate in many events of immunoendocrine interaction, characteristic of complex signaling networks. Thus, the signals from both the embryo and the mother during early embryo development converge around the actions of leukemia inhibitory factor (LIF), interleukin-1 (IL-1) and colony- stimulating factors (CSF), among others (1), supported by an adequate hormonal microenvironment and the recognition of the fetus by the maternal immune system. The embryo plays an active role in the modulation of the immunoendocrine activity during endometrial implantation, particularly by participating in (in the murine model) or enhancing (as in humans) the endometrial inflammatory reaction known as decidualization, thus regulating the production of maternal soluble factors that might be necessary for the invasion of the trophoblast for embryo lodging 
into the endometrium (2). These typical changes of decidualization can be induced with oil, air bubbles or concanavalin A and have also been observed in deciduomata (3) and ectopic pregnancies.

The decidual inflammatory reaction differs from the classical description of inflammation that occurs in response to injury. In particular, in decidua, the recruitment of leukocytes is mainly dominated by natural killer (NK) cells that in other contexts are recognized as cytotoxic cells which participate in the antitumoral and antiviral immune response. On the other hand, the transformation of the uterine stromal cells into decidual cells is demonstrated by prolactin production, increased IL-15 concentrations and activation of the arachidonic acid pathway. These factors enhance the decidual reaction in response to embryo invasion. In general, the decidual environment is able to regulate embryo differentiation, mainly the trophoectodermic layer, which is dependent on epithelial contact and extracellular matrix proteins from the stroma in addition to cytokines (4). These trophoectodermic/trophoblast cell subpopulations are producers of hormones and cytokines whose endocrine action can cross the maternal-fetal interface and directly influence the systemic physiological changes that characterize gestation.

In this intricate network of interactions cytokines act as intermediary links of the maternal-fetal relationship including the events related to decidualization, implantation, placentation, embryogenesis, and fetal growth. The aim of this review is to identify certain basic aspects of cytokine actions in maternal-fetal interaction and to suggest new possibilities for immunoendocrine regulation, which could be useful to understand some disorders of early pregnancy in humans and in other animal species.

\section{Early embryo development}

The establishment of the pre-implantatory embryo begins when the oocyte is fertilized by a spermatozoid. When the penetration of the male gamete into the oocyte is concluded, maternal and paternal pronuclei migrate to the center of the cell and the nuclear membranes fuse to allow the recombination of the genetic material. This is the first stage of embryo development, named zygote. Subsequently mitotic divisions of the zygote give rise to the stages of two, four and eight cells, until the morula (5). Then, the morula undergoes a process of cell polarization, where the outermost cells form the trophectoderm (external embryo layer). Simultaneously, the expression and activation of $\mathrm{Na}^{+} / \mathrm{K}^{+}$ATPase pumps transport sodium and water towards the interior of the morula that generates a cavity known as the blastocele (6). Meanwhile, a group of pluripotent cells denoted the inner cell mass is established in a pole of the embryo, between the trophectoderm and the blastocele. These changes give rise to the blastocyst, the final step of early embryo development.

Previous studies have provided information about the regulatory effects of the IL-1 system in human pre-implantatory embryos, as well as in embryos of other species (Table 1). The IL-1 system is composed of two agonists (IL- $1 \alpha$ and IL-1ß), one inhibitor designated IL-1 receptor antagonist (IL-1 ra), and two receptors known as IL-1 receptors type I (IL-1R tI) and type II. Interestingly, only IL-1R tI transduces a signal in response to IL-1 (7). Single blastomeres from human preimplantation embryos cultured in vitro express the mRNAs for IL-1ß, IL-1R tI and IL-1 ra (7). In bovine embryos the addition of IL- $1 ß$ after in vitro fertilization enhances the recovery of blastocysts under conditions of culture at high density (25-30 embryos/ drop) but not at low density (10 embryos/ drop). Thus, IL-1ß can modulate embryo development towards the blastocyst stage (8).

Human embryos cultured in vitro produce high concentrations of IL- $1 \alpha$ and IL- 
$1 \beta$ (>60 and $>80 \mathrm{pg} / \mathrm{mL}$, respectively), and the presence of these cytokines has been correlated with successful implantation after transfer to the uterine cavity (9). Kruessel et al. (10) demonstrated the expression of IL1B, IL-1R tI and IL-1 ra mRNA by RT-PCR in single-cell preimplantation mouse embryos. They did not detect the corresponding mRNAs at the stage of 2-cell-embryos, and only IL-1 ra was expressed at the 8-cellstage in a few embryos. Thus, the stage where IL-1ß, IL-1R tI and IL-1 ra could be detected was the compact morula stage. Therefore, this expression was increased by ongoing maturation and reached its highest level at the stage of hatched blastocyst (10).

The aim of assisted reproductive techniques and embryo culture is to obtain embryos of good quality with the ability to implant into the uterus. In this context, coculture systems of embryos on cell monolayers enhance early embryo development. Different types of cells such as uterine fibroblasts, human tubular cells, endometrial epithelial cells, and Vero cells (a cell line derived from green rhesus monkey kidney) have been used successfully. These monolayers produce embryotrophic factors that make it possible to obtain a higher yield of blastocysts. Some studies suggest that low

Table 1. Cytokines, prostaglandins, and growth factors implicated in embryo-endometrium interaction

\begin{tabular}{lll}
\hline Compounds & Biological actions & Reference \\
\hline LIF & Blastocyst implantation & Stewart et al., 1992 (40) \\
& Early embryogenesis as an embryotrophin & Lavranos et al., 1995 (16) \\
LIFR & Decidualization & Chen et al., 2000 (41) \\
IL-1 & Decidualization and placentation & Ni et al., 2002 (31) \\
& Successful implantation & Sheth et al., 1991 (9) \\
& Induces LIF production & Arici et al., 1995 (43) \\
& Induces COX-2 & Huang et al., 1998 (26) \\
IL-1 ra & Endometrial changes & McMaster et al., 1992 (23) \\
IL-1R tI & GM-CSF and CSF-1 production & Garcia-Lloret et al., 1994 (57) \\
EGF & Failure of implantation & Simon et al., 1994 (49) \\
& Embryo development & Kruessel et al., 1997 (10) \\
& Blastocyst differentiation & Kaye, 1997 (1) \\
& Angiogenic factor & Moller et al., 2001 (37) \\
HB-EGF & Trophoblastic differentiation & Kliman et al., 1990 (55) \\
& Induces LIF production & Arici et al., 1995 (43) \\
& Blastocyst growth, zona-hatching and & Das et al., 1994 (39) \\
IL-15 & trophoblast outgrowth & Leach et al., 1999 (36) \\
IGFBP-1 & Implantation reaction & Wang et al., 1994 (46) \\
TNF- $\alpha$ & Decidual marker & Okada et al., 2000 (19) \\
& Decidual marker & Dimitriadis et al., 2002 (20) \\
PGE2 & Endometrial changes & von Wolff et al., 1999 (24) \\
& GM-CSF and CSF-1 production & Garcia-Lloret et al., 1994 (57) \\
VEGF & Endometrial changes & Jabbour et al., 2003 (33) \\
GM-CSF & Multiple female reproductive failures & Lim et al., 1997 (28) \\
CSF-1 & Rifferentiation of NK cells & Reese et al., 2001 (29) \\
& Angiogenic factor & Linnemeyer et al., 1993 (35) \\
& Trophoblastic differentiation & Moller et al., 2001 (37) \\
& & Garcia-Lloret et al., 1994 (57) \\
& Garcia-Lloret et al., 1994 (57)
\end{tabular}

LIF = leukemia inhibitory factor; LIFR = LIF receptor; IL-1 = interleukin-1; IL-1 ra = IL-1 receptor antagonist; IL$1 \mathrm{R}$ tI = IL-1 receptor type I; EGF = epidermal growth factor; HB-EGF = heparin binding-EGF; IL-15 = interleukin-15; IGFBP-1 = insulin-like growth factor-binding protein 1; TNF- $\alpha=$ tumor necrosis factor alpha; $\mathrm{PGE}_{2}=$ prostaglandin $\mathrm{E}_{2} ; \mathrm{VEGF}=$ vascular endothelial growth factor; GM-CSF = granulocyte-macrophage colony-stimulating factor; CSF-1 = colony stimulating factor- 1 . 
concentrations of matrigel in embryo cultures enhance blastocyst formation and in vitro hatching (11). Matrigel is an extracellular matrix whose main structural components are proteins and growth factors that are also present in the endometrial extracellular matrix such as fibronectin, collagen type IV, heparan sulfate proteoglycans, entactin, nidogen, transforming growth factor $\beta$, fibroblast growth factor, and plasminogen tissue activator, among others. These compounds constitute a culture medium similar to the uterine microenvironment that is appropriate for embryo growth, viability, and for cytokine secretion. For example, the amount of the highly secreted IL- $1 \alpha$ in Quinn's HTF medium enriched with matrigel can be correlated with the embryo's ability to be implanted (11). Another explanation for this effect could be that these monolayers eliminate toxic metabolites generated by the embryo and mimic the environment of the oviduct (12). It is possible that matrigel increases IL- $1 ß$ and IL-1 ra and the expression of membrane receptors such as IL-1R tI, but this possibility has not yet been tested.

Before embryo implantation the blastocyst must escape from the zona pellucida (ZP), a thick extracellular cover composed of three glycoproteins: ZP1, ZP2, ZP3. This event is known as hatching, a dual process where the $\mathrm{ZP}$ is degraded by enzymes in both the trophectoderm and the uterus, facilitating blastocyst protrusion from the ZP. In humans, hatching occurs prior to implantation between the 5th and 7th day postovulation (13) and at 3.5 days post-mating in mice.

In humans and mice, hatching appears to be regulated by some cytokines, and the beneficial effect of LIF on embryo development, in vitro, has been very well documented (Table 1). LIF is a glycoprotein with a remarkable range of biological activities in various tissues; it is a highly glycosylated single chain polypeptide whose action is mediated through a LIF receptor-glycopro- tein 130-kDa complex (LIFR-gp130). Therefore, signal transduction and its biological activity require heterodimerization of these two low affinity, components LIFR and gp130, which generate a high-affinity binding site (14). The group of Tsai (15) concluded that culture media supplemented with recombinant human LIF (r-hLIF) enhances the rate of two-cell embryos reaching the stage of hatched blastocysts. Thus, the regulatory effect of LIF is associated with both early embryo development and viability: when early-stage embryos are cultured with $1,000 \mathrm{IU} / \mathrm{mL}$ of $\mathrm{r}-\mathrm{hLIF}$ the rate of blastocyst formation and trophoectodermic proliferation is enhanced (16).

\section{The peri-implantatory endometrial changes}

The endometrium is regulated by cyclic changes that modify the uterine microenvironment for embryo implantation. In humans the menstrual cycle has been described in two consecutive phases: proliferative or ovarian and secretory or luteal. This view of the cycle is supported by the hypothalamichypophysial-gonadal axis that gives rise to the ovarian and endometrial cycles. The beginning of the ovarian phase is mediated by the action of follicle-stimulating hormone which acts on several follicles that enter the maturation process during this cycle and on growth of dominant follicles. The granulosa and theca cells that surround the oocyte synthesize estrogens; these hormones influence the proliferation of the endometrial cells that is characteristic of the endometrial proliferative phase (17).

A positive feedback occurs in the hypothalamic-hypophysial-gonadal axis when estrogens are increased in mid-cycle, suddenly releasing luteinizing hormone and promoting ovulation. Granulosa and theca cells form the corpus luteum, an endocrine structure that produces progesterone and estrogen, hormones that are necessary to generate 
the phenotypic changes characteristic of the next phase of the endometrial cycle, i.e., the luteal or secretory phase. The action of these hormones is to transform the epithelial and stromal cells into a secretory phenotype, enhancing synthesis of vasoactive compounds such as prostaglandins (PGs) and cytokines related to the communication between mother and embryo $(17,18)$.

In the secretory phase, progesterone induces the morphological and phenotypic changes of the endometrial cells that are associated with the production of cytokines and inflammatory mediators (18). Changes occurring during the secretory phase in the stroma are known as decidualization, an inflammatory process characterized by the morphological transformation of the stromal cells from small spindle-shaped cells to large plump decidual cells. Decidual cells secrete prolactin, IL-15 and insulin-like growth factor-binding protein 1 (19-21). Spiral arteries and endometrial glands undergo characteristic changes during this phase: widening of the walls of the blood vessels and the muscle layer of the arteries and increase of the secretion of inflammatory mediators and cytokines by the endometrial gland cells (22).

Regulation of endometrial changes is not exclusively due to ovarian hormones: during the proliferative phase, for instance, mediators that induce an inflammatory process in the endometrium such as IL-1 (23), tumor necrosis factor alpha (TNF- $\alpha$ ) (24) and PGs are produced (25). This inflammatory process is characterized by edema and angiogenesis of the endometrial tissue, leukocyte recruitment and production of cytokines that could be participating in endometrium remodeling and blastocyst-endometrium interaction (23).

IL-1 production during the endometrial proliferative phase induces the release of vasoactive lipids such as PGs (26), whose synthesis is regulated by the cyclooxygenase (COX) enzyme, which presents two isoforms: COX-1 and COX-2. COX-1 is constitutively expressed and located in the gastric mucosa, while COX-2 presents an inducible expression related to inflammatory processes (27). The actions of PGs are not clearly understood, since COX-2 null mice showed a significant decrease of the weight of the uterus when decidualization was induced by intraluminal oil injection (28). The same observations were made when celecoxib and aspirin (selective inhibitors of COX-2 and COX-1, respectively) were used in wildtype mice (29). Based on these results, one of the mechanisms implicated in decidualization could be the action of $\mathrm{PGE}_{2}$ and prostacyclin $\left(\mathrm{PGI}_{2}\right)$ through production of cytokines and growth factors (30).

The enzymatic function of $\mathrm{COX}$ is to oxygenate arachidonic acid in cell membranes and generate PGH, which is the substrate used by tissue-specific isomerases producing different kinds of PGs (27). Using immunohistochemistry, Milne et al. (25) observed the production of PGE synthetase and $\mathrm{PGE}_{2}$ in the endometrial epithelium, stroma and endothelium during different phases of the menstrual cycle and also found that these compounds decrease during the late secretory phase. In the murine endometrium it has been documented that there is expression and production of both cytosolic and microsomal PGE synthetase isoforms produced by COX-1 and COX-2, respectively. mRNA expression and protein synthesis of these enzymes increase on day 5 post-ovulation, when murine endometrial decidualization begins $(31,32)$.

The pre-implantatory endometrium requires PGs during decidualization, especially for the vascular changes generated by its action as a vasodilator. Furthermore, new functions of $\mathrm{PGE}_{2}$ related to cell proliferation in the glandular epithelium have been described: epithelial cells stimulated with $\mathrm{PGE}_{2}$ significantly increased BrdU incorporation (33), indicating that glandular epithelial cell division is facilitated by $\mathrm{PGE}_{2}$, which may also exert these actions on other cell 
populations.

Another peculiarity of the early pregnant uterus is the recruitment of immune cells from peripheral blood, principally NK cells, which differentiate into another subpopulation distinct from circulating NK cells, i.e., uNK cells. The latter grow in size and granularity and, conversely, decrease their cytotoxic ability (34). Linnemeyer and Pollack (35) showed that NK cell differentiation could be induced by $\mathrm{PGE}_{2}$ in a concentration-dependent manner. Thus, $\mathrm{PGE}_{2}$ could be responsible for inducing these endometrial functions but the mechanisms for the induction of differentiation of these cells through $\mathrm{PGE}_{2}$ have not been elucidated.

Increased production of growth factors such as epidermal growth factor (EGF), heparin binding-epidermal growth factor (HBEGF) and vascular endothelial growth factor (VEGF) during both phases of the menstrual cycle has been observed in the endometrium $(36,37)$. VEGF and its receptor have been detected in endometrial cells and blood vessels during the proliferative and secretory phases with sustained protein expression of VEGF during both phases, but the number of receptors increases during the secretory phase (37). EGF has been found at high concentrations in epithelial cells, and its receptor is also increased in the secretory phase at the level of epithelia, stroma and blood vessels (37). HB-EGF production is enhanced in the endometrial stroma during both the proliferative and secretory phases in glandular epithelium and endometrial blood vessels (36). In this complex network a balanced cell production of inflammatory mediators and cytokines provides an adequate endometrial environment for interaction between the embryo and endometrium.

\section{Embryo implantation: blastocyst-endometrium interaction}

Embryo implantation can be defined as the process by which the embryo attaches to the endometrium (38), invades the decidualized stroma and reaches the maternal microvasculature (13). Hence, the endometrium requires structural modifications induced by progesterone and estrogens, pivotal changes that facilitate the three consecutive phases of implantation: apposition, attachment and invasion (38). This morphological and functional transformation of the endometrium around embryo implantation generates a period known as implantation window (13). In this context changes involve glycocalix modifications in the epithelial cells of the luminal surface, growth factor secretion and adhesion molecule expression related to blastocyst adherence and stromal invasion.

The murine blastocyst moves down the oviduct and falls into the uterine cavity and at that time it orientates its mural trophectoderm towards the epithelial surface. In humans, the hatched blastocyst opposes its polar trophectoderm to the surface of the epithelium on the fifth day of gestation, a process that occurs on the fourth day in mice. Das et al. (39) proposed that the implantation process begins as a kind of dialog between the embryo and the endometrium, prior to active penetration into the decidua. They observed a high level of HB-EGF by in situ hybridization in the luminal epithelium that surrounds the embryo, at the site of implantation, whereas HB-EGF was absent from pseudopregnant mice. Another finding related to blastocyst growth and hatching was obtained with HB-EGF, which enhances the cell number per blastocyst by almost $40 \%$ and leads to a 2-fold increase in the percentage of hatched embryos. Another cytokine, LIF, is necessary at this stage, as demonstrated in LIF-deficient females, whose embryos normally develop to the blastocyst stage but fail to implant (40). Implantation and normal pregnancy can occur when these embryos are transferred from LIF-deficient mothers to wild-type pseudopregnant recipients, or when an exogenous supply of LIF is provided to the LIF knock-out fe- 
males (41). Based on this approach, Vogiagis et al. (42) examined the estrous cycle and early pregnancy in ewes and observed an increase in immunoreactive LIF at the time of blastocyst hatching in the luminal and glandular epithelium. In humans, LIF is expressed throughout the cycle with a striking increase in LIF mRNA levels in the middle and late secretory phases, the period during which embryo implantation occurs (43). In the murine uterus, from days one to eight of pregnancy, the expression of LIFR and gp130 mRNAs was detected by in situ hybridization, reaching the highest levels on days 5 and 4, respectively (44). During early mouse embryogenesis the mRNAs of LIF, LIFR and gp130 are undetectable in 1- or 2-cell embryos, but are present in the blastocyst stage (45). This pattern of expression is strongly suggestive of a paracrine action of LIF between the embryo and the uterus during the implantation window.

Other evidence suggests that hormone actions are mediated by cytokines during the peri-implantation period. For instance, the nidatory surge of estrogens stimulates implantation in mice through the action of secondary factors such as LIF (41). Up-regulation of uterine LIF expression is coincident with high levels of estradiol $\left(\mathrm{E}_{2}\right)$ at ovulation and implantation. Thus, LIF can replace the nidatory effect of $E_{2}$ in ovariectomized female mice, inducing implantation and decidualization (41). Based on these findings, enhanced uterine expression of LIF could be mediated by a direct action of $\mathrm{E}_{2}$, but we rather postulate that such LIF expression can be induced by HB-EGF, which increases the level of LIF by stimulation of $\mathrm{E}_{2}(46)$ (Figure 1). In this context the action of LIF can be mediated also by other cytokines: an in vitro study with decidual human cells showed how the addition of $20 \mu \mathrm{L}$ rIL- 1 or $\mathrm{E}_{2}$ to the culture medium increased LIF production in a time-dependent manner (47). In another assay, Arici et al. (43) demonstrated that the addition of 0.01-10 ng EGF $\beta 1$ increased the amount of LIF mRNA in a concentrationdependent manner. These findings suggest that the function of LIF is mediated by the IL-1 system and by the action of the EGF family. We propose that LIF can exert its function in two ways: First, it may act in a synergistic manner with other cytokines on embryo hatching and attachment. Second, it may act on the endometrial epithelia in a paracrine manner to increase the expression of specific growth factors belonging to the IL-1 system and the EGF family that act on the trophectoderm promoting cell attachment and embryo development (Figure 1).

The IL-1 system has been considered to be relevant in regulating communication between the blastocyst and the endometrium. In the human and murine endometrium the IL- $1 \alpha$ and IL- $1 \beta$ mRNAs are expressed during the menstrual or estrous cycle. In hu-

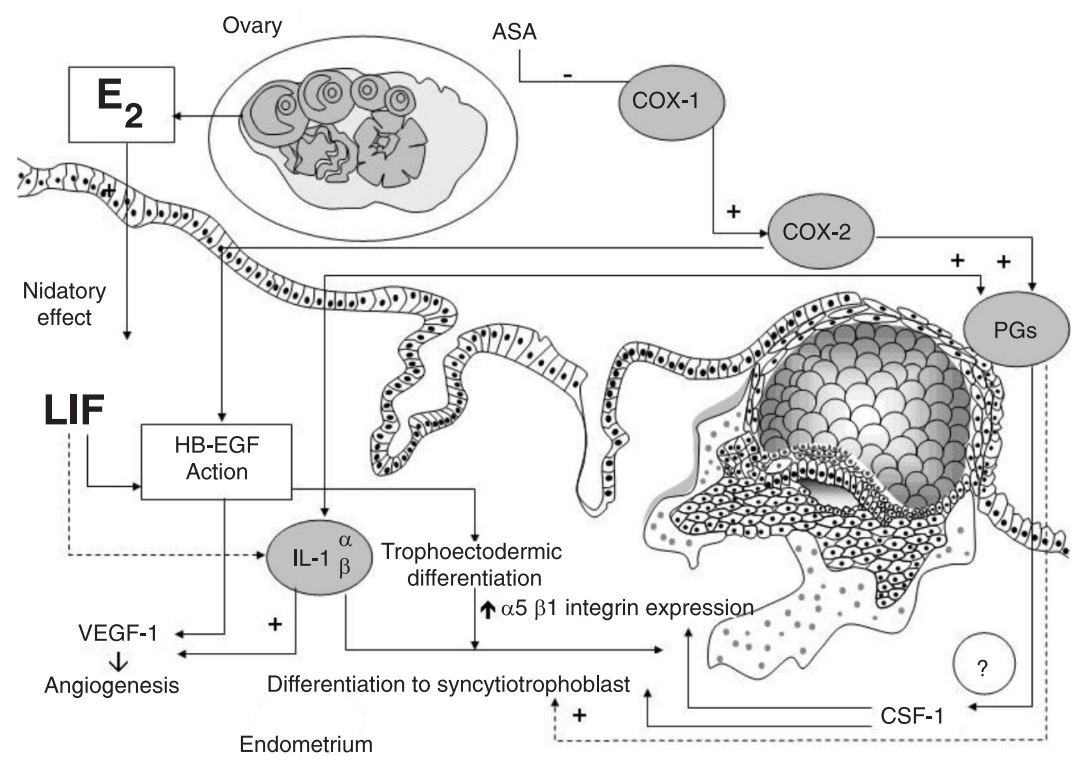

Figure 1. Model for the interaction between cytokines and mediators of inflammation associated with embryo implantation. The effect of estrogen $\left(E_{2}\right)$ is mediated by the nidatory action of leukemia inhibitory factor (LIF) in the endometrium, which is regulated by interleukin-1 (IL-1) and heparin binding-epidermal growth factor (HB-EGF). Both factors induce trophoblastic differentiation and angiogenesis through vascular endothelial growth factor (VEGF). Prostaglandin modulates IL-1 and possibly colony stimulating factor-1 (CSF-1) production. Both contribute to syncytiotrophoblast and anchoring cytotrophoblast differentiation. Aspirin (acetylsalicylic acid, ASA) acts in a selective manner on cyclooxygenase-1 $(C O X-1)$ and this could have an indirect effect on prostaglandin (PG) production by induction of COX-2. 
mans, IL-1 $\alpha$, IL-1ß, IL-1R tI, and IL- 1 ra are also expressed. This secretion of IL- $1 \alpha$, IL$1 \beta$ and IL- 1 ra changes in the endometrium according to the levels of steroid hormones, an event that is clearly visible when IL-1ß reaches a peak around the time of implantation. The IL-1R tI mRNA is expressed at increased concentrations in human endometrial epithelium during the luteal phase (48). In mice it was demonstrated that IL-1 ra interferes with embryonic attachment; IL-1 ra injected during the peri-implantation period induces failure of implantation (49).

The pre-ovulatory increase of 17- $ß$ estradiol stimulates both proliferation and differentiation of endometrial epithelial cells (17). In particular, 17- $\beta$ estradiol promotes an increase of apical protein density and a decrease of electronegative charges at the site of implantation. In some species, glycocalix changes are mediated by mucin 1 (MUC-1) expression on the epithelial surface in the endometrium, which overlaps putative binding sites of trophectoderm cells, mainly glycoconjugates and heparan sulfate-associated proteoglycans (50). For instance, MUC-1, a component of the apical glycocalix, is a large glycoprotein $(>200 \mathrm{kDa})$ with an extracellular domain rich in proline, serine and threonine residues. This protein is abundantly present on the apical glandular epithelial surface, and in the midsecretory implantation phase; it is also secreted into the gland lumen and in the uterine cavity. Overexpression of MUC-1 is able to induce steric inhibition of the adhesive properties of cultured cells and it is known that this might encourage metastatic spread. In mice and other species there is a distinctive reduction in MUC-1 protein expression during implantation, but not in humans (50), in whom down-regulation of this molecule may permit the expression of proteins such as heparan sulfate binding proteins that mediate embryo attachment.

Blastocyst attachment in humans occurs in the midsecretory phase. Aplin et al. (51) characterized the luminal epithelium at the time of implantation and observed a continuous staining pattern of MUC-1 between days 20 and 24 of the normal cycle (predicted period of the receptive phase). This means that the antiadhesive properties of MUC-1 detected in other species may not be valid in humans. These antiadhesive properties of MUC-1 have been shown to be due to the large extracellular domain, but it is clear from Aplin's work that immunoreactivity against sulfated glycosylation in apical epithelia is maintained during the proliferative phase and is increased in the early secretory phase. Thus, on day 20, apical staining with a 5D4 mAb, that recognizes keratan sulfate chains, was reduced in the luminal epithelium. Based on this observation, we propose that both the glycosylation with sulfated chains in the core protein of MUC-1 and the electronegativity of this molecule are altered during uterine receptivity. The antiadherence/ adherence balance is modulated in the endometrium by the attached embryo, which could induce indirect actions through enzymes such as metalloprotease and heparinase. In this way, the group of Simon (52) showed by immunofluorescence that MUC-1 was absent in endometrial epithelial cells subjacent and adjacent to the attached embryo, suggesting an active regulation by the embryo in the receptivity of the human endometrium (52).

On the other hand, synthesis of heparan sulfate in trophoectodermic cells increases 4- to 5-fold at the murine peri-implantation stage, which indicates that the embryo expresses proteoglycans necessary for adhesive interactions with the endometrium, such as syndecan and perlecan (53). The latter, as well as other proteoglycans (fibronectin and laminin) are expressed on the external surface of the trophectoderm and could interact with the extracellular matrix (54) in the decidualized endometrium. These compounds could also bind to members of the EGF family (amphiregulin, HB-EGF, among oth- 
ers, improving attachment and growth of the implantatory blastocyst.

\section{Effect of growth factors on trophoblast differentiation}

The decidua is thought to be a source of important growth factors and cytokines for placental development. Growth factors regulate PG synthesis in the endometrium, promoting the invasion, proliferation and changes related to endometrial vascular permeability. Enhanced PG production by the action of EGF has been shown in rat endometrial stromal cells. EGF acts through the phosphorylation of membrane proteins as a mitogen promoting DNA and RNA synthesis. As pregnancy progresses, the increased production of EGF enhances trophoblast differentiation (55).

Granulocyte-macrophage CSF (GMCSF) is produced by murine decidual cells in response to invasive trophoblast. Wegmann (56) documented the expression of GM-CSF, CSF-1, TNF- $\alpha$, IL-1, among other factors, in the murine placenta using a panel of cDNA probes. This pattern of cytokine expression may involve growth regulatory signals for the placenta (56). Garcia-Lloret et al. (57) demonstrated that both CSF-1 and GM-CSF stimulate the differentiation of the cytotrophoblast to the syncytiotrophoblast. This morphological differentiation was indirectly measured by the increased production of human placental lactogen (hPL) and human chorionic gonadotropin (hCG). Additionally, they found that GM-CSF and CSF-1 production was controlled by IL- 1 and TNF- $\alpha$ in fibroblasts derived from the villous stroma of the placenta. These cytokines could play an important role in the development and function of the human placenta (57).

Our group has studied the effect of aspirin on the production of GM-CSF and IL-3 by peripheral blood mononuclearcells (PBMC) and the effect of these cytokines on trophoblast differentiation. Briefly, PBMC were obtained from five men and five women and stimulated with aspirin, aspirin/phytohemagglutinin (PHA) and PHA or culture medium. The supernatants were used to evaluate the effect on the fusion of BeWo cells and the production of hPL and hCG as an indicator of syncytialization (Figures 2 and 3). We found that aspirin had no direct effect on GM-CSF and IL-3 production by PBMC, although the production of hPL and syncytialization over five or more nuclei were increased using PBMC supernatants obtained from women and stimulated with aspirin and aspirin/PHA (Bueno J, Cadavid L, Betancur L, Guzmán-Martinez C, Peña B and Cadavid Á, unpublished data) (Figure 3). We
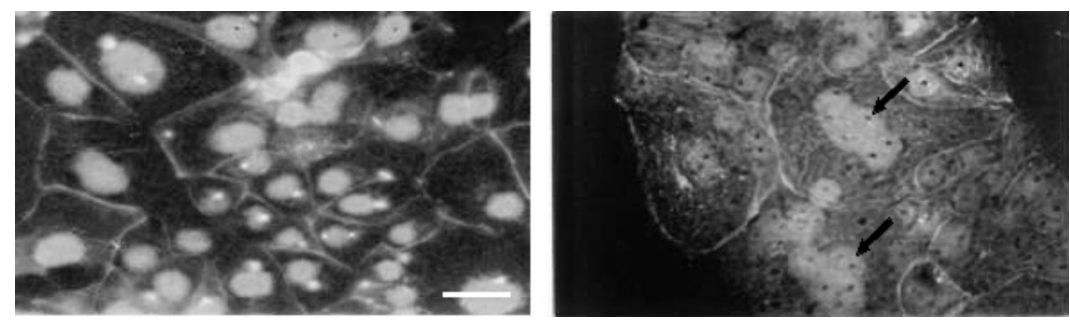

Figure 2. Cell fusion was quantitated by counting the number of nuclei, the number of cells, and the number of cells containing a single nucleus in each photographic field (black arrows). Cell fusion was grouped according to the number (2, 3, 4, 5, or more) of nuclei per cell. Scale bar $=15 \mu \mathrm{m}$.

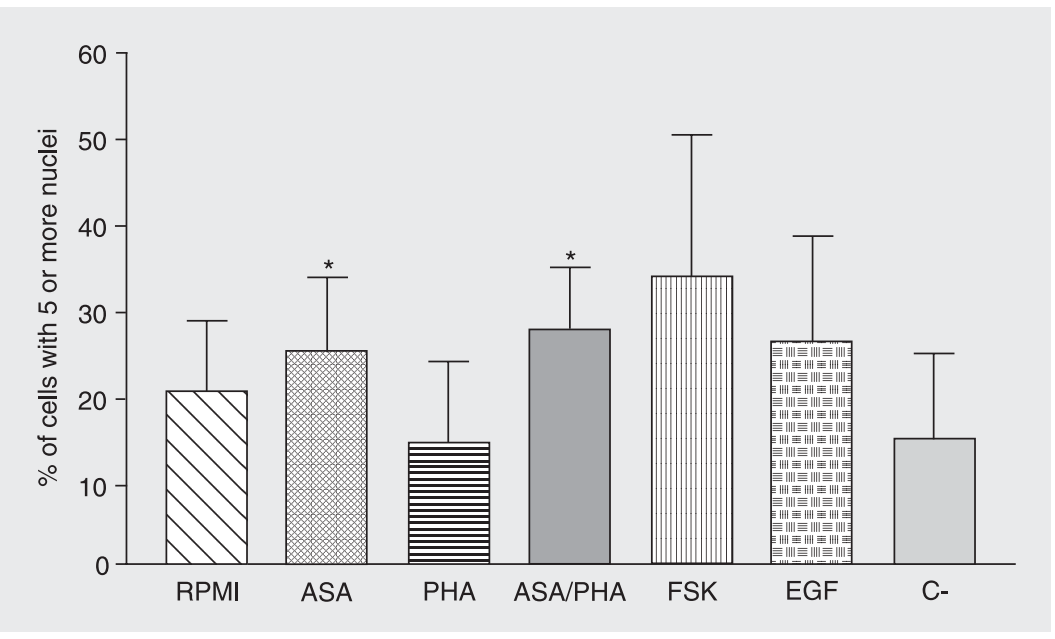

Figure 3. Correlation between cell fusion and human placental lactogen production in the group of women. The correlation between cell fusion and hormonal production was evaluated by the Pearson test $(P \leq 0.05)$. RPMI 1640 medium purchased from Sigma. $A S A=$ acetylsalicylic acid; PHA = phytohemagglutinin; FSK = forskolin; EGF = epidermal growth factor; $\mathrm{C}-$ = negative control. 
conclude that the trophoblast differentiation induced by the effect of aspirin cannot be correlated with GM-CSF or IL-3 production. Perhaps other cytokines could be induced by aspirin to improve syncytialization. Moreover, since it is possible that aspirin can induce some effect on growth factor secretion by the trophoblast per se, we have performed some experiments in which aspirin directly induced an increase in trophoblast syncitialization, maybe through the autocrine action of growth factors produced by these cells.

\section{Therapeutical approaches}

A recently developed r-hLIF (or emfilermin) will be tested in human clinical trials. The experimental evidence in animals has demonstrated the physiological role of LIF in implantation, but the implications of the use of r-hLIF in human reproductive medicine are not clear. LIF has a wide spectrum of action and therefore has been used for many diseases; e.g., in a phase I study including patients with advanced lung cancer, a biological effect of r-hLIF was demonstrated by the increase of blood progenitor cells, C-reactive protein levels, and hemopoietic recovery after chemotherapy (58). On the basis of reports from animal and human studies, we agree with the statement made by SERONO in its website (59) that LIF could be used as a safe therapeutical alternative and as a potent inducer of human embryonic implantation.

For the adaptive modifications of the endometrial environment, steroid hormones are a key factor, particularly $E_{2}$ that exerts its actions on the production of LIF in the endometrium. On this basis, we propose that the nidatory surge of LIF during the implantation window could induce a change of the electronegative charges on the epithelial surface and the remodeling of the glycocalix to home the embryo. It is possible that glycosylation of MUC-1 changes these electronega- tive charges produced by keratan sulfate chains in the glycoprotein structure and finally enhances the exposure of heparan sulfate-binding proteins in the epithelium. LIF could play an important role in these modifications, improving the attachment of the embryo to the endometrium and ensuring trophoectodermic differentiation towards the fibronectin-binding phenotype influenced by HB-EGF.

We believe that the differentiation of NK cells and other endometrial cells such as stromal cells may be induced by $\mathrm{PGE}_{2}$. At the same time this could amplify the production of other cytokines that participate in decidualization and embryo implantation. Furthermore, PG production could also act on trophoblast proliferation and differentiation. The therapeutic use of aspirin prior to implantation could be useful for successful pregnancy because it has been demonstrated that aspirin can inhibit the action of COX-1 in platelets and up-regulate COX-2 production from other sources. This stimulus potentiates $\mathrm{PGE}_{2}$ production that could finally enhance trophoblast differentiation and increase the vasodilatory action necessary for adequate blood perfusion of the embryo.

Although therapeutic strategies based on cytokine targets have been used in many models and in diseases such as cancer, the results reported are not completely conclusive in clinical trials, maybe due to the complexity of the cytokine network implicated in each disease. Alternative pathways could be activated when a cytokine is blocked or when it is used as an inducer of a specific immune response. New therapeutical approaches must focus on drugs able to induce the production of several cytokines, in order to maintain an adequate profile of cytokines that support the desired response. Aspirin could be one such compound: it is an ancient drug with unexplored actions on cytokine production, for instance on IL-3 production by mononuclear cells. We did not establish what kind of cytokine modulate the syncy- 
tialization of the BeWo cells throughout acetylsalicylic acid or what kind of mediators, e.g., $\mathrm{PGE}_{2}$ or leukotrienes, were indirectly implicated in this immune modulation but it is possible that more than one cytokine was involved in these effects. The probable induction by aspirin of cytokine production by trophoblastic cells cannot be ruled out in this context: the trophoblast has an important role in the maternal-fetal dialogue and aspirin could modulate bidirectionally this interaction.

In other animal models such as the pig, the immunomodulator effect of $\mathrm{PGE}_{2}$ has been shown on growth factor and cytokine production by lymphocytes during gestation (60). In humans, this same action on the lymphocytes and other cells such as NK cells could be stimulated by $\mathrm{PGE}_{2}$ and could induce the production of cytokines such as those of the CSF family. Thus, implantation and trophoblast invasion could be mediated by CSF-1, GM-CSF and other cytokines produced by cells localized in the decidua.

Finally, IL-1ß induces COX-2 synthesis (26) and LIF production (47) by human endometrial stromal cells and in decidual cells in vitro. These results strongly suggest that PGs may control blastocyst implantation through a paracrine effect of the IL-1. On the other hand, they could suggest a synergistic effect of both IL-1 and LIF on blastocyst implantation (Figure 1).

Detailed information about the cellular and molecular mechanisms associated with the action of cytokines on stromal cells and on the blastocyst during implantation will have a significant impact on the understanding of the fundamental causes of implantation failure. Moreover, the participation of the trophoblast in the paracrine dialogue between mother and fetus must be understood in more detail due to its pivotal action on embryo and fetus development.

\section{Acknowledgments}

The authors are grateful for the help and encouragement of Professors Angela Cadavid (coordinator of the Reproduction Group at the University of Antioquia), Juan Guillermo Maldonado (coordinator of the CENTAURO Group at the University of Antioquia), AnneLise Haenni (from the Institute Jacques Monod - France), and Jorge Ossa (from the University of Antioquia).

\section{References}

1. Kaye PL. Preimplantation growth factor physiology. Rev Reprod 1997; 2: 121-127.

2. Kimber SJ. Cell biology of implantation and plancentation. Introduction. Semin Cell Dev Biol 2000; 11: 61-65.

3. Shaw TJ, Murphy CR. Leucocyte involvement in lectin-induced deciduomata formation. Cell Biol Int 1995; 19: 577-584.

4. Pollheimer J, Knofler M. Signalling pathways regulating the invasive differentiation of human trophoblasts: a review. Placenta 2005; 26 (Suppl A): S21-S30.

5. Cooke S, Quinn P, Kime L, Ayres C, Tyler JP, Driscoll GL. Improvement in early human embryo development using new formulation sequential stage-specific culture media. Fertil Steril 2002; 78: 12541260.

6. Watson AJ. The cell biology of blastocyst development. Mol Reprod Dev 1992; 33: 492-504.

7. Krussel JS, Simon C, Rubio MC, Pape AR, Wen Y, Huang HY, et al. Expression of interleukin-1 system mRNA in single blastomeres from human preimplantation embryos. Hum Reprod 1998; 13: 22062211.
8. Paula-Lopes FF, de Moraes AA, Edwards JL, Justice JE, Hansen PJ. Regulation of preimplantation development of bovine embryos by interleukin-1beta. Biol Reprod 1998; 59: 1406-1412.

9. Sheth KV, Roca GL, al-Sedairy ST, Parhar RS, Hamilton CJ, alAbdul JF. Prediction of successful embryo implantation by measuring interleukin-1-alpha and immunosuppressive factor(s) in preimplantation embryo culture fluid. Fertil Steril 1991; 55: 952-957.

10. Kruessel JS, Huang HY, Wen Y, Kloodt AR, Bielfeld P, Polan ML. Different pattern of interleukin-1 beta- (IL-1 beta), interleukin-1 receptor antagonist- (IL-1ra) and interleukin-1 receptor type I- (IL-1R tl) mRNA-expression in single preimplantation mouse embryos at various developmental stages. J Reprod Immunol 1997; 34: 103120.

11. Lazzaroni L, Fusi FM, Doldi N, Ferrari A. The use of Matrigel at low concentration enhances in vitro blastocyst formation and hatching in a mouse embryo model. Fertil Steril 1999; 71: 1133-1137.

12. Ouhibi N, Hamidi J, Guillaud J, Menezo Y. Co-culture of 1-cell mouse embryos on different cell supports. Hum Reprod 1990; 5 : 737-743. 
13. Norwitz ER, Schust DJ, Fisher SJ. Implantation and the survival of early pregnancy. N Engl J Med 2001; 345: 1400-1408.

14. Senturk LM, Arici A. Leukemia inhibitory factor in human reproduction. Am J Reprod Immunol 1998; 39: 144-151.

15. Tsai HD, Chang CC, Hsieh YY, Lo HY, Hsu LW, Chang SC. Recombinant human leukemia inhibitory factor enhances the development of preimplantation mouse embryo in vitro. Fertil Steril 1999; 71: 722725.

16. Lavranos TC, Rathjen PD, Seamark RF. Trophic effects of myeloid leukaemia inhibitory factor (LIF) on mouse embryos. J Reprod Fertil 1995; 105: 331-338.

17. Chabbert-Buffet $\mathrm{N}$, Bouchard $\mathrm{P}$. The normal human menstrual cycle. Rev Endocr Metab Disord 2002; 3: 173-183.

18. Rider V. Progesterone and the control of uterine cell proliferation and differentiation. Front Biosci 2002; 7: d1545-d1555.

19. Okada S, Okada H, Sanezumi M, Nakajima T, Yasuda K, Kanzaki H. Expression of interleukin-15 in human endometrium and decidua. Mol Hum Reprod 2000; 6: 75-80.

20. Dimitriadis E, Salamonsen LA, Robb L. Expression of interleukin-11 during the human menstrual cycle: coincidence with stromal cell decidualization and relationship to leukaemia inhibitory factor and prolactin. Mol Hum Reprod 2000; 6: 907-914.

21. King A. Uterine leukocytes and decidualization. Hum Reprod Update 2000; 6: 28-36.

22. Fazleabas AT, Strakova Z. Endometrial function: cell specific changes in the uterine environment. Mol Cell Endocrinol 2002; 186 : 143-147.

23. McMaster MT, Newton RC, Dey SK, Andrews GK. Activation and distribution of inflammatory cells in the mouse uterus during the preimplantation period. J Immunol 1992; 148: 1699-1705.

24. von Wolff M, Classen-Linke I, Heid D, Krusche CA, Beier-Hellwig K, Karl C, et al. Tumour necrosis factor-alpha (TNF-alpha) in human endometrium and uterine secretion: an evaluation by immunohistochemistry, ELISA and semiquantitative RT-PCR. Mol Hum Reprod 1999; 5: 146-152.

25. Milne SA, Perchick GB, Boddy SC, Jabbour HN. Expression, localization, and signaling of $\mathrm{PGE}(2)$ and EP2/EP4 receptors in human nonpregnant endometrium across the menstrual cycle. J Clin Endocrinol Metab 2001; 86: 4453-4459.

26. Huang JC, Liu DY, Yadollahi S, Wu KK, Dawood MY. Interleukin-1 beta induces cyclooxygenase-2 gene expression in cultured endometrial stromal cells. J Clin Endocrinol Metab 1998; 83: 538-541.

27. Majerus PW. Prostaglandins: critical roles in pregnancy and colon cancer. Curr Biol 1998; 8: R87-R89.

28. Lim H, Paria BC, Das SK, Dinchuk JE, Langenbach R, Trzaskos JM, et al. Multiple female reproductive failures in cyclooxygenase 2deficient mice. Cell 1997; 91: 197-208.

29. Reese J, Zhao X, Ma WG, Brown N, Maziasz TJ, Dey SK. Comparative analysis of pharmacologic and/or genetic disruption of cyclooxygenase-1 and cyclooxygenase-2 function in female reproduction in mice. Endocrinology 2001; 142: 3198-3206.

30. Kimber SJ. Leukaemia inhibitory factor in implantation and uterine biology. Reproduction 2005; 130: 131-145.

31. Ni H, Sun T, Ding NZ, Ma XH, Yang ZM. Differential expression of microsomal prostaglandin $\mathrm{E}$ synthase at implantation sites and in decidual cells of mouse uterus. Biol Reprod 2002; 67: 351-358.

32. Ni H, Sun T, Ma XH, Yang ZM. Expression and regulation of cytosolic prostaglandin $\mathrm{E}$ synthase in mouse uterus during the peri-implantation period. Biol Reprod 2003; 68: 744-750.

33. Jabbour HN, Boddy SC. Prostaglandin E2 induces proliferation of glandular epithelial cells of the human endometrium via extracellular regulated kinase 1/2-mediated pathway. J Clin Endocrinol Metab 2003; 88: 4481-4487.

34. Pollack SB, Linnemeyer PA. Natural killer cells in the nonpregnant murine uterus. Nat Immun 1996; 15: 34-40.

35. Linnemeyer PA, Pollack SB. Prostaglandin E2-induced changes in the phenotype, morphology, and lytic activity of IL-2-activated natural killer cells. J Immunol 1993; 150: 3747-3754.

36. Leach RE, Khalifa R, Ramirez ND, Das SK, Wang J, Dey SK, et al. Multiple roles for heparin-binding epidermal growth factor-like growth factor are suggested by its cell-specific expression during the human endometrial cycle and early placentation. J Clin Endocrinol Metab 1999; 84: 3355-3363.

37. Moller B, Rasmussen C, Lindblom B, Olovsson M. Expression of the angiogenic growth factors VEGF, FGF-2, EGF and their receptors in normal human endometrium during the menstrual cycle. Mol Hum Reprod 2001; 7: 65-72.

38. Cavagna M, Mantese JC. Biomarkers of endometrial receptivity - a review. Placenta 2003; 24 (Suppl B): S39-S47.

39. Das SK, Wang XN, Paria BC, Damm D, Abraham JA, Klagsbrun M, et al. Heparin-binding EGF-like growth factor gene is induced in the mouse uterus temporally by the blastocyst solely at the site of its apposition: a possible ligand for interaction with blastocyst EGFreceptor in implantation. Development 1994; 120: 1071-1083.

40. Stewart CL, Kaspar P, Brunet LJ, Bhatt H, Gadi I, Kontgen F, et al. Blastocyst implantation depends on maternal expression of leukaemia inhibitory factor. Nature 1992; 359: 76-79.

41. Chen JR, Cheng JG, Shatzer T, Sewell L, Hernandez L, Stewart CL. Leukemia inhibitory factor can substitute for nidatory estrogen and is essential to inducing a receptive uterus for implantation but is not essential for subsequent embryogenesis. Endocrinology 2000; 141: 4365-4372.

42. Vogiagis D, Fry RC, Sandeman RM, Salamonsen LA. Leukaemia inhibitory factor in endometrium during the oestrous cycle, early pregnancy and in ovariectomized steroid-treated ewes. J Reprod Fertil 1997; 109: 279-288.

43. Arici A, Engin O, Attar E, Olive DL. Modulation of leukemia inhibitory factor gene expression and protein biosynthesis in human endometrium. J Clin Endocrinol Metab 1995; 80: 1908-1915.

44. Ni H, Ding NZ, Harper MJ, Yang ZM. Expression of leukemia inhibitory factor receptor and gp130 in mouse uterus during early pregnancy. Mol Reprod Dev 2002; 63: 143-150.

45. Nichols J, Davidson D, Taga T, Yoshida K, Chambers I, Smith A. Complementary tissue-specific expression of LIF and LIF-receptor mRNAs in early mouse embryogenesis. Mech Dev 1996; 57: 123131.

46. Wang XN, Das SK, Damm D, Klagsbrun M, Abraham JA, Dey SK. Differential regulation of heparin-binding epidermal growth factorlike growth factor in the adult ovariectomized mouse uterus by progesterone and estrogen. Endocrinology 1994; 135: 1264-1271.

47. Sawai K, Matsuzaki N, Okada T, Shimoya K, Koyama M, Azuma C, et al. Human decidual cell biosynthesis of leukemia inhibitory factor: regulation by decidual cytokines and steroid hormones. Biol Reprod 1997; 56: 1274-1280.

48. Simon C, Mercader A, Frances A, Gimeno MJ, Polan ML, Remohi J, et al. Hormonal regulation of serum and endometrial IL-1 alpha, IL-1 beta and IL-1ra: IL-1 endometrial microenvironment of the human embryo at the apposition phase under physiological and supraphysiological steroid level conditions. J Reprod Immunol 1996; 31 : 165-184.

49. Simon C, Frances A, Piquette GN, el Danasouri I, Zurawski G, Dang $W$, et al. Embryonic implantation in mice is blocked by interleukin-1 
receptor antagonist. Endocrinology 1994; 134: 521-528.

50. Braga VM, Gendler SJ. Modulation of Muc-1 mucin expression in the mouse uterus during the estrus cycle, early pregnancy and placentation. J Cell Sci 1993; 105 ( Pt 2): 397-405.

51. Aplin JD, Meseguer M, Simon C, Ortiz ME, Croxatto $H$, Jones CJ. MUC1, glycans and the cell-surface barrier to embryo implantation. Biochem Soc Trans 2001; 29: 153-156.

52. Meseguer M, Aplin JD, Caballero-Campo P, O'Connor JE, Martin JC, Remohi J, et al. Human endometrial mucin MUC1 is up-regulated by progesterone and down-regulated in vitro by the human blastocyst. Biol Reprod 2001; 64: 590-601.

53. Carson DD, Tang JP, Julian J. Heparan sulfate proteoglycan (perlecan) expression by mouse embryos during acquisition of attachment competence. Dev Biol 1993; 155: 97-106.

54. Smith SE, French MM, Julian J, Paria BC, Dey SK, Carson DD. Expression of heparan sulfate proteoglycan (perlecan) in the mouse blastocyst is regulated during normal and delayed implantation. Dev Biol 1997; 184: 38-47.

55. Kliman HJ, Feinberg RF, Haimowitz JE. Human trophoblast-en- dometrial interactions in an in vitro suspension culture system. Placenta 1990; 11: 349-367.

56. Wegmann $\mathrm{T}$. The role of cytokine cross-talk in preventing abortion. Res Immunol 1990; 141: 185-188.

57. Garcia-Lloret MI, Morrish DW, Wegmann TG, Honore L, Turner AR, Guilbert LJ. Demonstration of functional cytokine-placental interactions: CSF-1 and GM-CSF stimulate human cytotrophoblast differentiation and peptide hormone secretion. Exp Cell Res 1994; 214 : 46-54.

58. Gunawardana DH, Basser RL, Davis ID, Cebon J, Mitchell P, Underhill $C$, et al. A phase I study of recombinant human leukemia inhibitory factor in patients with advanced cancer. Clin Cancer Res 2003; 9: 2056-2065.

59. SERONO site. http://www.seronousa.com/news/archives/June3003. jsp. Accessed March 16, 2006.

60. Li TK, Fox BS. Effect of prostaglandin E2 (PGE2) on IL-3/granulocyte-macrophage colony-stimulating factor production by $\mathrm{T}$ helper cells. Mode of stimulation and presence of costimulation can determine response to PGE2. J Immunol 1993; 150: 1680-1690. 